\title{
1 Multisensory binding is driven by the strength of stimulus correlation
}

3 Aaron R. Nidiffer ${ }^{* 1,2}$, Ramnarayan Ramachandran ${ }^{2,3,4,6}$, Mark T. Wallace ${ }^{2,3,4,5,6}$

4

$5 \quad{ }^{1}$ Department of Biomedical Engineering, University of Rochester, Rochester, NY, USA

$6 \quad{ }^{2}$ Department of Hearing and Speech Sciences, Vanderbilt University, Nashville, TN, USA

$7 \quad{ }^{3}$ Vanderbilt Brain Institute, Vanderbilt University, Nashville, TN, USA

$8 \quad{ }^{4}$ Department of Psychology, Vanderbilt University, Nashville, TN, USA

$9 \quad{ }^{5}$ Department of Psychiatry, Vanderbilt University, Nashville, TN, USA

$10{ }^{6}$ Vanderbilt Kennedy Center, Vanderbilt University, Nashville, TN, USA

*Correspondences: aaron.nidiffer@rochester.edu

\section{Abstract}

Our perceptual system is adept at exploiting sensory regularities to better extract information about our environment. One clear example of this is how the sensory and multisensory systems can utilize consistency to group sensory features into a perceptual object and segregate objects from each other and background noise. Leveraging tenets of object-based attention and multisensory binding, we sought whether this ability scaled with the strength of that consistency. We presented participants with amplitude modulated (AM) auditory and visual streams and asked them to detect imbedded orthogonal, near-threshold frequency modulation (FM) events. We modulated the correlation of the streams by varying the phase of the visual AM. In line with a previous report, we first observed peak performance that was shifted from $0^{\circ}$. After accounting for this, we found that across participants discriminability of the FM event linearly improved

25 with correlation. Additionally, we sought to answer a question left dangling from our previous report as to the possible explanation for the phase shift. We found that phase shift correlated with auditory and visual response time differences, but not point of subjective simultaneity, suggesting differences in sensory processing times may account for the observed phase shift. These results suggest that our perceptual system can bind multisensory features across a spectrum of temporal correlations, a process necessary for multisensory binding in complex environments where unrelated signals may have small errant correlations. 
Introduction

Our conscious perception depends crucially on our ability to appropriately piece together separate features of events in the environment. Our perceptual system effortlessly accomplishes this across auditory and visual domains in processes called auditory scene analysis (Bregman, 1990) and visual grouping (Wertheimer, 1923). Each unisensory system relies on a set of cues (e.g., consistency in the spectral, spatial, and temporal structure) that influence the binding of features into objects (Carlyon, 2004; Feldman, 2003; Shinn-Cunningham, 2008). One cue common to both unisensory modalities, temporal correlation (Bizley \& Cohen, 2013; Lee \& Blake, 1999), has been shown to be a robust driver of binding features across modalities (Bizley, Maddox, \& Lee, 2016). Indeed, correlation across time is a property of multisensory signals that originate from a common source, such as the articulatory and acoustic features of speech (Chandrasekaran et al., 2009; Plass et al., 2019). The multisensory system utilizes these temporal correlations to enhance our perception (Grant \& Seitz, 2000; Maddox et al., 2015; Parise \& Ernst, 2016; Parise, Spence, \& Ernst, 2012; Parise et al., 2013; Plass et al., 2019).

Despite demonstrating the importance of temporal correlation on perception, studies have been largely limited to a restricted set of correlations-perfectly correlated (or matched) and uncorrelated (cf., Parise et al., 2013). Because of this, it is unclear whether our perceptual system evaluates stimuli for the presence of correlation or evaluates the strength of correlation between stimuli. This distinction may become more important in the context of multiple competing stimuli (e.g., the cocktail party problem; Cherry, 1953) since, for example, ambiguous mouth movements can have errant correlations with unrelated acoustic speech envelopes presence of correlation could be one strategy to select between competing streams, thus mitigating this problem.

Recently, we found that behavioral performance does vary with the strength of the correlation imbedded in dynamic audiovisual (AV) stimuli (Nidiffer et al., 2018), showing that the brain does perform a computation on the strength of audiovisual correlation. However, in that study, participants were making judgements on the presence of the correlated feature, amplitude modulation. It has been suggested that to unambiguously dissociate multisensory integration from binding, judgements should be made on a stimulus feature orthogonal to the

2 feature that induces the binding (Bizley, Maddox, et al., 2016). This assertion is rooted in 
63 principles of object-based attention (Blaser, Pylyshyn, \& Holcombe, 2000; Desimone \&

64 Duncan, 1995; Shinn-Cunningham, 2008; Treisman, 1998) where attention, when directed

65 toward a specific feature of an object, enhances other features of that object.

In the current study, we first leveraged these principles detailed below to determine whether our previous findings extend to multisensory binding. Participants were asked to make judgements on a brief spectral feature (frequency modulation) while we modulated the correlation of the temporal structure (amplitude modulation) in an audiovisual stream. We first measured a discrepancy between unisensory signals previously reported to be individualized across participants. Accounting for these individual differences, we found that behavioral performance on the orthogonal feature does change with temporal correlation in a linear fashion, though to a lesser degree than when judgements are made on the correlated feature. We conducted two further experiments to probe the relationship between phase shift and other multisensory quantities, speeded detection and simultaneity judgement, to garner some understanding of the processes involved in determining phase shift. We find that phase shift is correlated to the differences in unisensory detection.

\section{Results}

Multisensory binding is modulated by stimulus correlation proportional to the strength of correlation

In the first experiment, participants detected near threshold frequency modulation (FM) events imbedded in amplitude modulated (AM) audiovisual streams (Figure 1). The phase of the visual AM was adjusted across conditions, thereby changing the correlation between the auditory and visual streams. If the AM correlation is inducing binding of the audiovisual streams, we expect the change in stimulus correlation to induce changes in the perception of the FM event. Figure 2 shows discriminability (d') data from two participants plotted across visual phase. There is a clear cyclic pattern in these data (Figure 2a). Moreover, there seems to be a phase shift whereby the best discrimination did not occur when the auditory and visual AM streams perfectly overlapped, a finding that was thoroughly tested in our previous report (Nidiffer et al., 2018). 

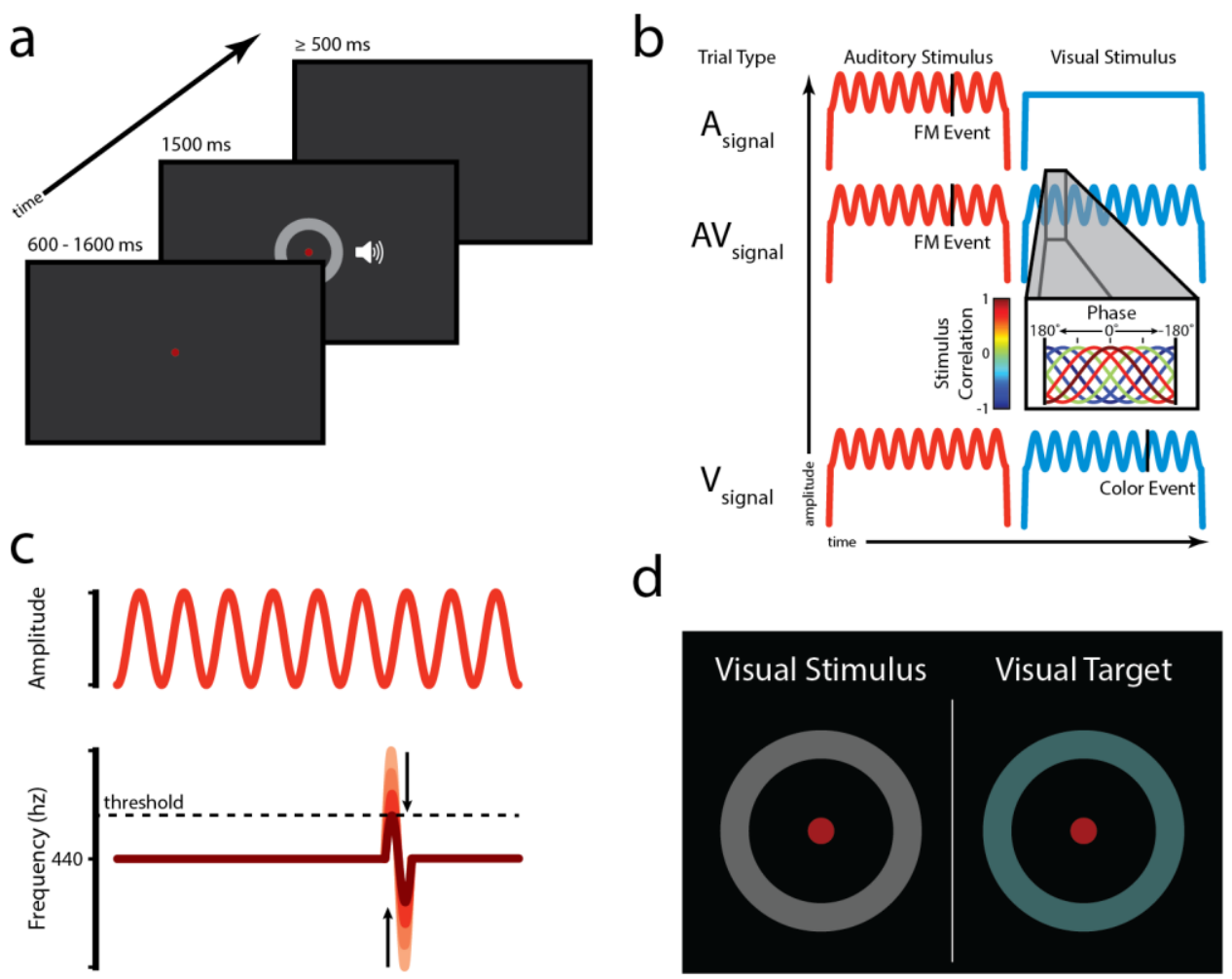

Figure 1. Stimulus and task. a: Participants fixated a red dot at the center of the display. After a variable time, an amplitude modulated (AM) audiovisual stimulus lasting $1.5 \mathrm{~s}$ appeared. Participants were asked to report the presence of a frequency (c) or color (d) deviation target. b: AM could be present in the auditory stimulus (by modulating the intensity) or both auditory and visual stimuli (by modulating the luminance). Targets consisting of a frequency deviation or color deviation could be present at $1 \mathrm{~s}$ after stimulus onset. During audiovisual AM with the frequency deviation, the phase of the visual modulation was varied to generate a range of stimulus correlations (inset).c: The auditory stimulus consisted of a single pure tone of $440 \mathrm{~Hz}$ which could be briefly $(100 \mathrm{~ms})$ frequency modulated (FM). FM depth was adjusted to the threshold of each participant via 3-down-1-up staircase procedure prior to the main experiment. $d$ : The visual stimulus was a grey ring set upon a black background. During a visual event, its color would briefly (100 ms) change to blue.

In an effort to account for this phase shift, we fit these data to a sine wave with phase as a free parameter (see methods) to measure the deviation of the peak from zero. Because this deviation is consistent with a timing difference between unisensory systems and highly individualized as previously shown, measuring individuals' deviation is necessary for calculating each participant's perceptual correlation. Previously, we have shown that this phase shift can be measured by iteratively fitting phase-shifted correlation matrices ( 8 phases $\times 5$ frequencies) to discriminability matrices. However, since we only measured discriminability at one frequency in 
the current study, we sought to compare these two methods using data from the previous study.

110 Discriminability data from the first frequency in those data were fit using the same procedure

111 shown in Figure 2a. These two methods produce comparable results (Figure 2b, abscissa shows

112 data from Figure 2e in Nidiffer et al., 2018) when considering participants that had reliable 113 estimates of their phase shift. Not only are they strongly correlated $(\mathrm{r}=0.95, \mathrm{p}=0.02)$, but their 114 values are very consistent (slope $=0.92, \mathrm{CI}:[0.73,1.13])$. Thus, we concluded that fitting a sine 115 wave on data from one frequency produces a robust measure of phase shift.
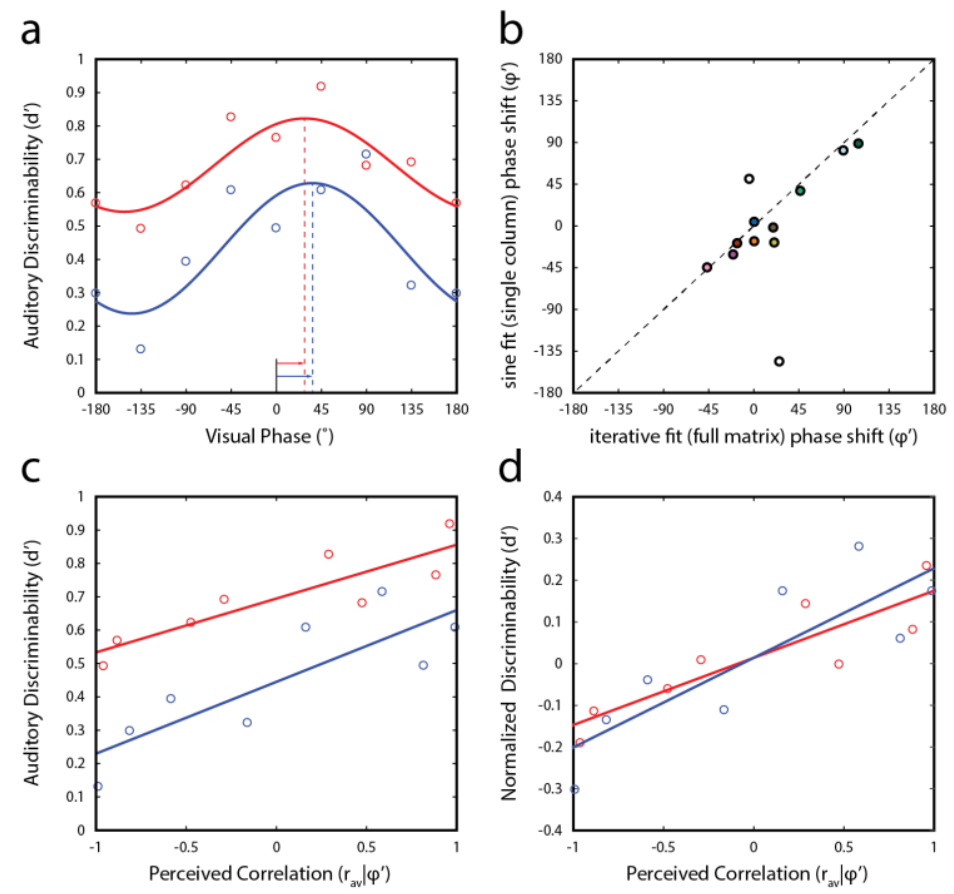

116

117

118

119

120

121

122

123

124

125

126

127

128

129

Figure 2. Individual data transformation. a: Discriminability (d')—a measure of sensitivity—of the frequency event was calculated and is shown plotted against the visual phase. Each phase maps onto a different stimulus correlation. A cyclic relationship between visual phase and auditory discriminability is evident. As shown previously, best performance is not aligned to the best correlated stimuli in the environment $\left(0^{\circ}\right)$ but is instead shifted along phase (arrows). We fit this curve with a sine wave and extracted the phase shift parameter to recompute correlation in perceptual space. b: Using data from our previous report, we compared phase shift values derived from the previously described iterative correlation fit of the full discriminability matrix to a sine fit of the first column of the same matrix. Both methods return very similar values for phase shift. c: Auditory discriminability plotted against perceived audiovisual correlation which accounts for the phase shift in (a). Discriminability is clearly modulated by correlation in a linear manner. Although this relationship is evident across the sample, individual participants performed at different levels. d: Normalized discriminability was calculated in order to account for individual differences in performance. The values of $d$ ' were centered for each participant and is shown plotted here as a function of perceived audiovisual correlation. 

correlations based on these new envelopes were computed for each participant. Participants' discriminability is plotted against these stimulus correlations in Figure 2c. On average, the correlation between these measures was strong $(r=0.40 \pm 0.2$; mean \pm std. dev. $)$, however, due to the lack of data points, only two of 17 participants showed significant improvement. Therefore, we decided to pool participant data to observe group effects. First, to account for participants who are performing at different levels, we normalized (centered) each participants' discriminability (see methods; Figure 2d). Consistent with previous research, discriminability of the FM feature was significantly improved by temporal correlation between the auditory and visual AM feature. In line with our hypothesis, this relationship was positive and linear such that stronger correlations were associated with better discriminability (Figure 3a; $r=0.32 \mathrm{CI}$ : [0.18 0.57], $\mathrm{p}<0.0001)$. As expected, when pooling participants, the correlation coefficient decreased.

142 However, the observed average and the pooled normalized correlations were significantly better than a null distribution that was permuted from shuffled data

a

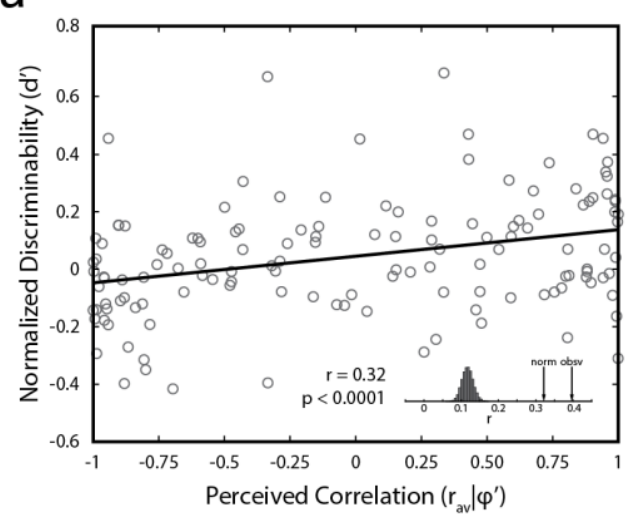

b

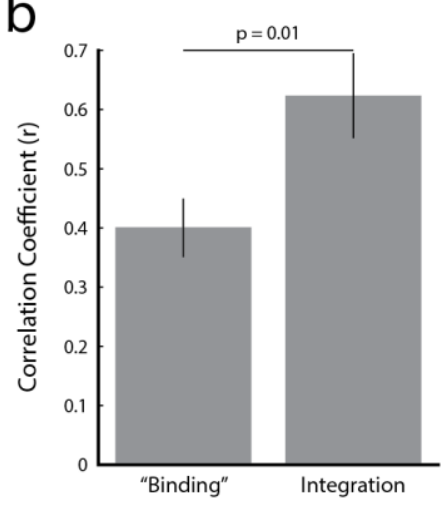

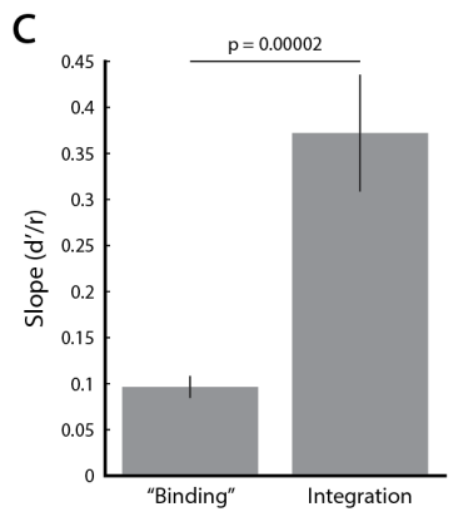

Figure 3. Group binding data. a: Normalized discriminability across all participants is significantly correlated to perceived temporal correlations. The observed and normalized correlation (inset, arrow) is significantly higher than a permuted null distribution (see methods). b: Correlation coefficients (mean \pm s.e.m.) from each participant nonnormalized data in the current study ("Binding") and a previous study (Integration) are not significantly different, indicating similar effect sizes between the two data sets. c: Slope (mean \pm s.e.m.) of the line of best fit to nonnormalized data in the current and previous study are different, indicating a lower dependence on correlation in the current study.

In our previous report (Nidiffer et al., 2018), participants detected AM in similar stimuli. In that study, participants similarly showed a linear increase in discriminability of the AM, an effect that can't be unambiguously attributed to the binding of the stimuli (Bizley, Maddox, et 
155 al., 2016). In both studies, the dependence on correlation was measured by finding the slope of

156 the best fitting line between stimulus correlation and discriminability. The effect sizes for

157 individual participants (non-normalized) were significantly different between the current $(\mathrm{r}=$

$1580.40 \pm 0.20)$ and previous investigation $\left(r=0.62 \pm 0.25 ; t_{27}=-2.65, p=0.013\right.$; figure $\left.3 b\right)$, the

159 dependence of binding on correlation (slope $=0.09 \pm 0.04$ ) was significantly smaller than the

160 dependence of integration on correlation (slope $=0.37 \pm 0.22 ; t_{27}=-5.15, \mathrm{p}=2.0 \times 10^{-5}$; figure $3 \mathrm{c}$ ).

161 The difference across the most positive and negative correlation $\left(\Delta \mathrm{d}^{\prime}=0.18\right)$ is comparable to a

162 study that used only two extremes of similarity ( $\Delta \mathrm{d}^{\prime} \approx 0.2$; Maddox et al., 2015).

163 AM phase shift is related to unisensory temporal processing

164 Previously, we found that individuals show unique differences in their perception of

165 multisensory correlation that could be accounted for by lagging one modality with respect to the

166 other (Nidiffer et al., 2018). We hypothesized that the resultant measure, phase shift, is related

167 to individual differences in unisensory processing speeds. There are several known phenomena

168 that are related to these differences between unisensory processing. First, response times in a

169 speeded detection task have been shown to be different for auditory and visual stimuli and lead to

170 different asynchronies that result in peak multisensory gain across participants (Hershenson,

171 1962). We had an a priori expectation that measures taken from an RT task would correlate with

172 phase shift due to the similarity in the tasks and probably similarity in neural architecture

173 underlying the two behaviors (Bizley, Jones, \& Town, 2016). Second, point of subjective

174 simultaneity and binding window width which accounts for temporal differences in our

175 perception of audiovisual synchrony and relate to both sensory differences and differences in

176 propagation in the environment (Zampini et al., 2005). Due to the difference between this task,

177 synchrony judgement, and our task, detection, we expected the correlation between these

178 measures and ours to be weak at best. Two additional experiments were conducted in order to

179 quantify measures of unisensory and multisensory temporal processing and to relate them to

180 phase shifts obtained in the current experiment. 

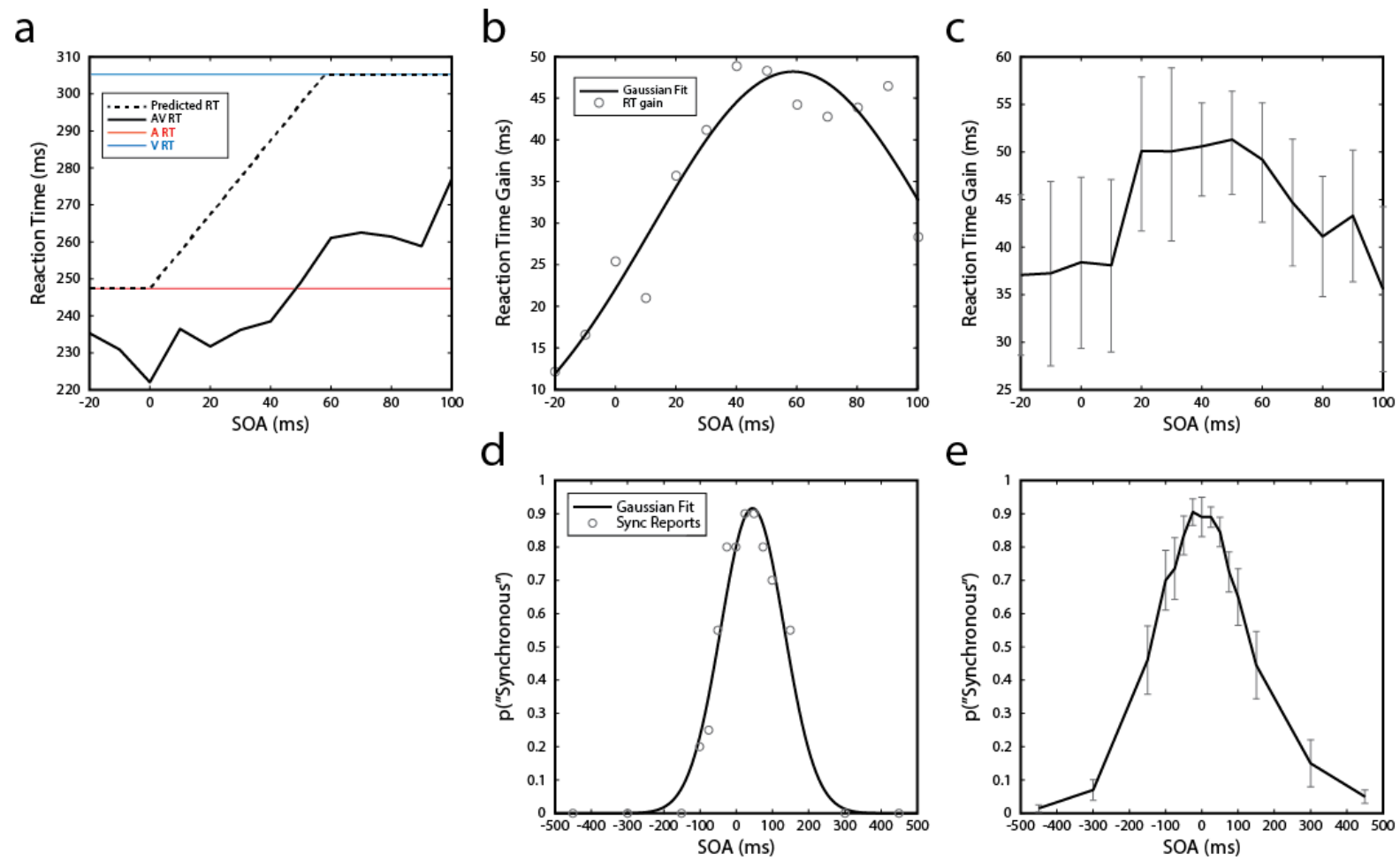

182 Figure 4. Individual and group response time and simultaneity judgement data. a: Response time data from an individual participant in Experiment 2. Auditory (red line), visual (blue line) and audiovisual (solid black line) response times are plotted against SOA. Unisensory response times are plotted across SOAs for illustrative purposes. Auditory response time is faster than visual response times, which was typical for most participants. A prediction (dashed line) of audiovisual response time computed from the unisensory data while accounting for lag imposed by SOA is presented for comparison with empirical audiovisual response times. Audiovisual response times typically were faster than this prediction. b: Response time gain for a single participant from (a) was calculated by subtracting obtained audiovisual response times from their predictions. This curve was fit with a Gaussian function for each participant. c: Response time gain (mean \pm s.e.m.) across all participants show gains across typical SOAs. d: Simultaneity judgement data from the same participant in (a) is shown across SOAs. This curve was fit with a Gaussian function for each participant. e: Simultaneity judgements (mean \pm s.e.m.) across all participants exhibit typical temporal binding windows and the typical rightward shift.

In Experiment 2, participants responded as quickly as possible to auditory, visual, and audiovisual stimuli with a button press. Audiovisual stimuli were presented a different stimulus onset asynchronies (SOAs), including objective synchrony. Figure 4a shows response times for auditory (red line), visual (blue line), and audiovisual (black line) stimuli in a single subject. The multisensory response times across SOAs were subtracted from a prediction (dashed line) based on the unisensory response times while accounting for stimulus lag for that condition (Figure 4b- 
c). These curves were fit to a gaussian function $\left(R^{2}=0.59 \pm 0.22\right)$. From these data, unisensory RT difference (Figure 4a, blue minus red lines) and the SOA producing peak RT gain (Figure 4b, mean parameter of Gaussian fit, $\mu$ ) were computed for each participant. Experiment 3 consisted of a simultaneity judgement task. Audiovisual stimuli were presented with a larger range of SOAs and participants were asked to report whether they perceived the stimuli to be synchronous or asynchronous, emphasizing accuracy over speed. The probability of reporting 206 synchrony was calculated across SOAs (Figure 4d-e). These curves were fit to the same Gaussian

207 function $\left(\mathrm{R}^{2}=0.95 \pm 0.03\right.$; Figure $\left.4 \mathrm{~d}\right)$ and we extracted measures of the point of subjective simultaneity (mean, $\mu$ ) and window width (2×standard deviation, $\sigma$ ).

Each of these measures, unisensory RT difference, peak RT gain, PSS, and window width are plotted against phase shift in Figure 5. The difference between auditory and visual response times $(50.1 \pm 34.9 \mathrm{~ms})$ along with the delay corresponding to peak RT gain $(42.6 \pm 48.6$ ms) were in line with those reported previously (Hershenson, 1962). Although the mean PSS (7.4 $\pm 29.3 \mathrm{~ms})$ was slightly lower that what has been previously reported (Zampini et al., 2005), the distributions overlap considerably. In comparison, window size in the current study (429.9 \pm 203.1$)$ was considerably larger than was reported in that study (Zampini et al., 2005).

217 and longer stimuli are known to produce wider temporal windows (Vroomen \& Stekelenburg, 218 2011; Wallace \& Stevenson, 2014). When comparing to phase shift, neither temporal window measures, PSS and window width, were correlated with phase shift. However, both RT difference $(r=0.63, p=0.031)$ and peak RT $(r=0.62, p=0.032)$ were significantly correlated

221 with phase shift, indicating that they likely share a common process. 
a

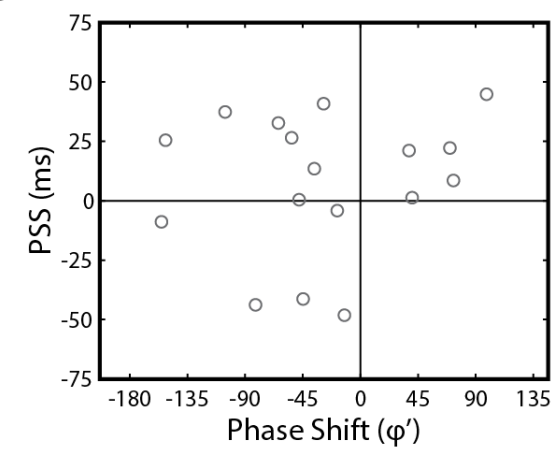

C

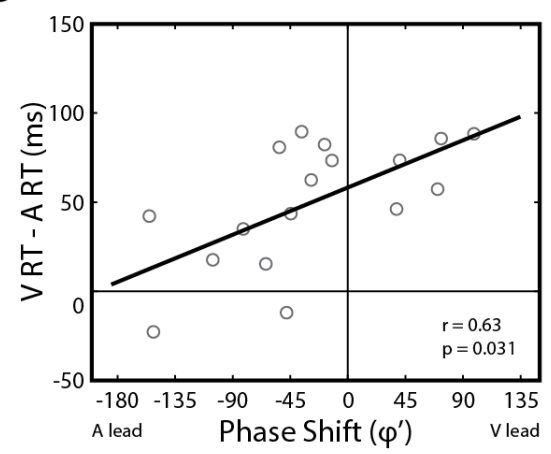

b

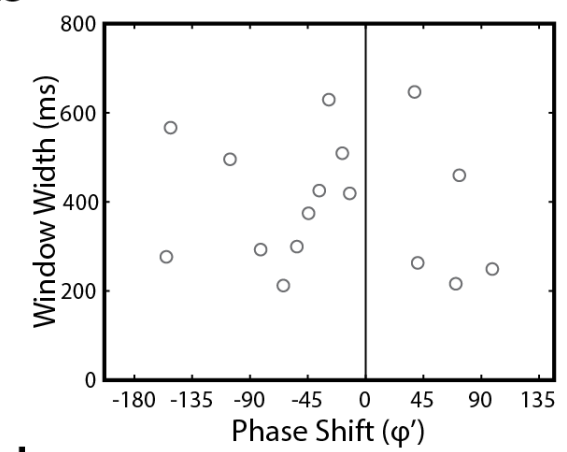

d

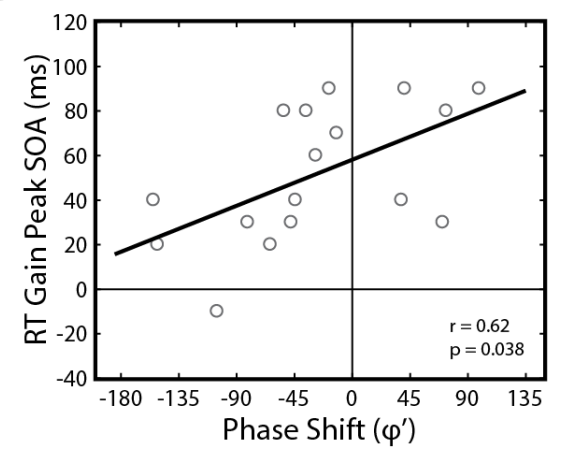

Figure 5. Phase shift correlates with unisensory response time differences. a: Point of subjective simultaneity (PSS)

228 is plotted against phase shifts obtained in Experiment 1. b: Temporal binding window width is plotted against phase 229 shifts obtained in Experiment 1. c: Unisensory RT differences are correlated phase shifts obtained in Experiment 1.

$230 \mathrm{~d}$ : RT peak gain SOA is plotted against phase shifts obtained in Experiment 1.

231

232

233

234

235

236

237

238

239

240

241

\section{Discussion}

Temporal correlation has been shown to influence multisensory processing in a variety of ways. Detection is improved in both simple stimuli (Nidiffer et al., 2018) and speech (Grant \& Seitz, 2000), cue combination is statistically optimal (Parise et al., 2012), spatial and temporal segregation is more difficult (Chuen \& Schutz, 2016; Jack \& Thurlow, 1973; Parise et al., 2013; Vatakis \& Spence, 2007), and selective attention is improved (Maddox et al., 2015). Previously, we showed that multisensory benefits scale with the magnitude of the correlation (Nidiffer et al., 2018), which could potentially provide a means for selection of appropriate signals in challenging environments. Here, we build upon these findings, using tenets of object-based attention (Desimone \& Duncan, 1995; Shinn-Cunningham, 2008), to show that multisensory binding occurs proportional to the level of correlation. 
The fact that multisensory interaction scales with the strength of correlation requires the neural computation of the degree of similarity between environmental signals. Further, that this process can influence the perception of other features is evidence that multisensory binding is dependent on that computation. Given that our environment is composed of auditory and visual signals such as speech that span a range of correlations (Chandrasekaran et al., 2009), this computation may underly the appropriate association of those signals in complex acoustical environments where a binary determination of correlation may be insufficient.

Griffiths and Warren (2004) proposed four principles that define perceptual objecthood, specifically for auditory objects. Among those principles is the notion that information in the sensory world related to an object is separable from other sensory information. Temporal correlation (i.e., coherence) has been shown to be a strong cue for the binding and segregation of sound sources (Elhilali et al., 2009; O’Sullivan, Shamma, \& Lalor, 2015; Teki et al., 2013). In short, we can form an auditory object based on the temporal correlation of its features and segregate that object from an uncorrelated background. Our ability to form a perceptual object and segregate a background is enhanced by a visual stimulus that is also correlated to (Maddox et al., 2015). Since multisensory binding is proportional to the strength of the correlation, a natural extension of the current work is to directly test whether auditory stream segregation is proportionally enhanced by a visual stimulus in the same way by leveraging stimulus competition in a stream segregation task while manipulating the strength of the correlation of a visual stream.

Previously, it was demonstrated that rhythmic audiovisual stimuli with the strongest correlations (i.e., those with the same frequency and phase) do not necessarily drive the best behavioral performance (Nidiffer et al., 2018). Instead, performance was found to be shifted along the phase dimension. A likely candidate for the neural explanation for this phenomenon is differences in stimulus processing time between the sensory systems, especially those involving the entrainment of oscillations. Although there is no direct evidence of such a process, there are several pieces of tangential evidence. First, unisensory entrainment is different across individuals (Henry \& Obleser, 2012; Simon \& Wallace, 2017), suggesting some variability in the entrainment process that might also manifest across modalities. Second, entrainment timing is malleable under conditions involving audiovisual onset timing differences (Kösem, Gramfort, \& Van Wassenhove, 2014). Here we provide further support for this hypothesis in showing that phase shift is related to unisensory response time differences. 
Although detection speed and oscillatory entrainment are largely separate phenomena, it

274 is possible that their differences across the sensory systems are a manifestation of general timing

275 differences. It is unclear whether the phase relationship discussed here is preserved across

276 frequencies or is simply a timing difference between the sensory systems. Further, whether this

277 phenomenon has any bearing on audiovisual binding of more complex, arrhythmic stimuli

278 remains to be seen. One study relating stimulus correlation to multisensory integration found

279 that correlation lead to optimal cue combination, irrespective of onset timing (i.e., cross-

280 correlation; Parise et al., 2012). However, the stimuli used in that study were arrhythmic

281 auditory and visual events, rather than continuous, rhythmic fluctuations, and the authors

282 reported no analysis concerning sensory delay.

283

284

285

286

287

288

289

290

291

292

293

294

295

296

297

298

299

300

301

302

303

Our findings and those of others (Atilgan et al., 2018; Maddox et al., 2015), suggest that audiovisual temporal correlation enhances the discrimination of a frequency feature, suggest one pathway in which visual speech enhances the discrimination of acoustic speech. The cortical representation of an auditory stream, both speech and non-speech, is enhanced by a congruent visual stream (Atilgan et al., 2018; Crosse, Butler, \& Lalor, 2015). Visual congruence also enhances the representation of orthogonal frequency features embedded in the auditory stream (Atilgan et al., 2018). Interestingly, this is likely an oversimplification of how our perceptual system exploits the correlation between features of visual and acoustic speech; a recent finding (Plass et al., 2019) showed that human listeners have learned to associate horizontal (but not vertical) expansion and contraction of the mouth with rising and falling frequency sweeps, respectively (but not vice-versa). Frequency features and their cortical representation are important for speech perception (Elliott \& Theunissen, 2009; Mesgarani \& Chang, 2012) and frequency discrimination ability has been linked to speech perception (Nan et al., 2018).

\section{Materials and Methods}

\section{Participants}

17 individuals (age $=25.4 \pm 4.9,10$ females) participated in the current study. All participants reported normal or corrected-to-normal vision and normal hearing and were right handed. The study was conducted in accordance with the declaration of Helsinki, and informed written consent was obtained from all participants. All procedures were approved by the Vanderbilt University Institutional Review Board. When applicable, participants were given monetary compensation or course credit for participation. 
304

305

306

307

308

309

310

311

312

313

314

315

316

317

318

319

320

321

322

323

324

325

326

327

328

329

330

331

332

333

334

Experiment 1

Apparatus and stimuli

All stimuli were generated in MATLAB (The MathWorks, Inc., Natick, MA) and presented using PsychToolbox version 3(Brainard, 1997; Kleiner et al., 2007). Auditory stimuli were digitized at $44.1 \mathrm{kHz}$, and presented through calibrated open-back circumaural headphones (Sennheisser HD480). Visual stimuli were centered about a red fixation dot at the center of a dark $\left(0.15 \mathrm{~cd} / \mathrm{m}^{2}\right)$ viewing screen (Samsung Sync Master 2233rz, $120 \mathrm{~Hz}$ refresh rate).

The auditory stimulus was a single frequency $(440 \mathrm{~Hz})$ tone presented at moderate level (48 dB SPL, A-weighted). Visual stimuli consisted of a moderately bright ring $\left(24 \mathrm{~cd} / \mathrm{m}^{2}\right.$; inner diameter: $1.8^{\circ}$, outer diameter: $3.6^{\circ}$ visual angle) over a black background. Both stimuli were presented simultaneously, lasted $1.5 \mathrm{~s}$, and were gated by a linear $10 \mathrm{~ms}$ onset and offset ramp. Stimulus timing was confirmed with a Hameg 507 oscilloscope, photodiode, and microphone. The amplitude of each stimulus, $y$, was modulated over time, $t$, such that

$$
y(t)=[1+m(t)] \times c(t)
$$

where

$$
m(t)=M \times \cos \left(2 \pi f_{a m} t+\varphi_{0, j}\right)
$$

and $c(t)$ is the time series of the carrier stimulus (auditory: tone; visual: ring). The form of the amplitude modulation (AM) signal $m(t)$ is defined by a modulation depth $M$ which represents the amplitude of the modulation signal as a proportion of the amplitude of the carrier signal, modulation frequency $f_{a m}$ in $\mathrm{Hz}\left(f_{a m}=6\right)$, and starting phase $\varphi_{0, j}$ in degrees. The auditory stimulus could contain a frequency modulation (FM) event such that

$$
c(t)=\cos \left(2 \pi f_{c} t+\frac{f_{\Delta}}{f_{f m}} \sin \left(2 \pi f_{f m} t\right)\right)
$$

Where $f_{c}$ is the frequency of the tone in $\mathrm{Hz}\left(f_{c}=440\right), f_{\Delta}$ is the deviation of the $\mathrm{FM}$ in $\mathrm{Hz}$, and $f_{\text {frm }}$ is the frequency of the $\mathrm{FM}$ in $\mathrm{Hz}\left(f_{f m}=10\right)$. A FM event was implemented using the $f_{m}$ mod () in MATLAB and briefly (100 ms, one full FM cycle) occurred 1 second after stimulus onset so that it occurred at exactly the same phase of the AM to obviate any behavioral dependence on AM phase (Henry \& Obleser, 2012).

The AM signal was always present in the auditory channel with modulation depth set to $\mathrm{M}=0.5$ and starting phase set so that the modulation begins at the trough $\left(\varphi_{0, j}=0^{\circ}\right)$. Visual AM could be present $(M=0.5)$ or absent $(M=0)$. Thus, this configuration produced four separate conditions based on a factorial design included Auditory AM (aAM, no visual AM) vs 
Audiovisual AM (avAM) and FM event present (go trials) vs no FM event (catch trials). Visual modulation during audiovisual go trials occurred with various starting phases $\left(\varphi_{0}=\{-135,-90,-\right.$ $\left.45,0,45,90,135,180^{\circ}\right\}$, with $\left.\varphi_{o_{j}} \in \varphi_{0}\right)$. Because we were interested in the interactions between auditory and visual stimuli on an orthogonal feature, but the task could be carried out with only the auditory stimuli, we included and additional visual target condition (go trial) to ensure participants were observing the visual stimuli. In this condition, which contained AM in both auditory and visual streams, the color of the visual stimulus changed gradually from its base color $(\mathrm{RGB}=\{100,100,100\})$ to blue-green $(\mathrm{RGB}=\{60,100,100\})$ and back over $100 \mathrm{~ms}$. The visual and auditory target never occurred in the same trial.

Procedure

Participants were seated comfortably inside an unlit WhisperRoom ${ }^{\mathrm{TM}}$ (SE 2000 Series) with their forehead placed against a HeadSpot ${ }^{\mathrm{TM}}$ (University of Houston Optometry) with the forehead rest locked in place such that a participant's primary eye position was centered with respect to the fixation point at the center of the viewing screen. Chinrest height and chair height were adjusted to the comfort of the participant.

Prior to the main experiment, each participant completed a 3-down 1-up staircase procedure to obtain an estimate of their FM deviation $\left(f_{\Delta}\right)$ threshold. For the staircase procedure, on a given trial (Figure 1a), a red fixation dot appeared at the center of the screen. Participants were instructed to fixate the dot for its entire duration. After a variable time $(0.6-1.6$ seconds), the auditory stimulus was presented. The FM event was presented at random for each trial. Participants were instructed to report the presence of the FM event (described as a "frequency deviant") after the stimulus presentation by pressing "1" on the number pad of a computer keyboard if the modulation was present or pressing " 0 " if the modulation was absent. The $f_{\Delta}$ decreased after three successive correct responses and increased after one incorrect response. At the beginning of each staircase, the step size was set to increase or decrease $f_{\Delta}$ by $5 \mathrm{~Hz}$. After two reversals (correct to incorrect response or incorrect to correct response), step size was reduced to $2 \mathrm{~Hz}$. Finally, after eight reversals, step size became $1 \mathrm{~Hz}$ in order to arrive at an accurate estimate threshold. Each staircase terminated after 20 reversals. Threshold was determined to be the average of the modulation depth at the last 10 reversals.

Instructions included an example of a stimulus with FM at the initial starting modulation depth $\left(f_{\Delta}=30\right)$ and an example of a stimulus with no FM. So that there was no ambiguity in 
cases where the first trial did not include a modulation signal, participants were informed that the first trial would have the same modulation depth as the example if present. To control for "runs" of trials with no modulation during the staircase (which could result in erroneously low threshold estimates), a sequence of two trials containing no modulation was always followed by a trial with modulation. At the conclusion of the staircase, the experimenter visually inspected the staircase for its typical asymptotic form and had participants repeat the procedure if necessary.

After the staircase procedure, participants completed two blocks of the main experiment. On a given trial, the central red fixation dot appeared. After a variable period, one of five types (I-V) of stimuli were presented. Each block consisted of (I) 20 avAM go trials per visual phase with auditory events (160 trials total), (II) 23 avAM go trials with a visual event, and (III) 11 aAM go trials with auditory events. Additionally, there were (IV) 24 avAM catch trials ( 10\% of total trials) and (V) 11 aAM catch trials ( 5\% of total trials). Participants were asked to judge the presence of an auditory or visual event with a button press as quickly and accurately as possible. Participants were instructed to press " 1 " on the number pad if an auditory or visual event occurred during a trial and " 0 " if it did not. Responses were counted if they occurred within 3 seconds of the event. Each block contained in total 229 trials, was identical in trial composition, and were presented in a pseudorandom order. Breaks were offered frequently (every 50 trials) to prevent fatigue. Participants were shown examples of each stimulus prior to the first block. Analysis

Discriminability ( $d$; a measure of sensitivity) for each of the 8 audiovisual conditions and two unisensory conditions was computed from the relative frequencies of the respective responses,

$$
d_{i}{ }^{\prime}=z\left(H_{i}\right)-z(F)
$$

where $H_{i}$ is the proportion of hits ("1" $\mid$ FM stimulus) for the $i^{\text {th }}$ condition, $F$ is the proportion of false alarms ("1" | no FM stimulus) from the corresponding catch trial condition, and $z$ is the inverse of the normal distribution function (MATLAB's norminv function).

Because we have previously shown that perception of rhythmic audiovisual stimuli is shifted across phase uniquely in each participant, we fit d' data for the audiovisual AM conditions to the function

$$
\widehat{d}^{\prime}=\mathrm{a} \times \cos \left(\varphi_{0, j}+\varphi^{\prime}\right)+b
$$

where $\varphi_{0, j}$ is the starting phase of the visual stimulus. The fitting returns parameters $\varphi^{\prime}$, the phase shift, and $a$ and $b$, which influence the magnitude and shift of the function, respectively. We 
recalculated the correlations using the phase shift parameter as described previously (Nidiffer et al., 2018) and these phase-shifted correlations were used to measure the dependence of behavior on stimulus correlation. To normalize d', which was on different scales across participants, we subtracted the $b$ parameter from individual data, thus centering the data while preserved the d' units. All data were pooled across participants and Pearson correlation was computed for stimulus correlation and discriminability. We tested this value against a permuted null distribution by shuffling the labels of the visual phase on AV trials before reanalysis that followed the same procedure used for non-shuffled data.

Experiment 2 and 3

Apparatus and stimuli

The stimuli were brief (166 ms) and consisted of the same visual ring described above and a frozen token of broadband auditory noise. The visual stimulus was presented about a fixation dot that remained on the screen, uninterrupted, for the entirety of the experiments. The noise was generated by MATLAB's randn() function and presented diotically at moderate level (48 $\mathrm{dB}$ SPL, A-weighted). The amplitude envelope of both stimuli was modulated as described in Experiment 1, but because the duration was 166ms, only one trough-to-trough cycle of AM was present. Thus, the stimuli did not appear to flutter, but had the same envelope characteristics. Auditory and visual stimuli could be present individually (only in Experiment 2) or together during a trial. When presented together, they were presented synchronously or with a stimulus onset asynchrony ( $\mathrm{SOA}$ ) by delaying one stimulus relative by a short interval. For Experiment $2 \mathrm{SOA}=\{ - \pm 10, \pm 20,30,40,50,60,70,80,90$, and 100ms $\}$. For Experiment 3, $\mathrm{SOA}=\{ \pm 25, \pm 50, \pm 75, \pm 100, \pm 150, \pm 300, \pm 450 \mathrm{~ms}\}$. Negative values indicate that the visual stimulus occurred after the auditory stimulus.

Procedure

Experiments 2 and 3 occurred between the two blocks of Experiment 1 and their order was randomized across participants. Both experiments took 12-15 minutes to complete. Participants were seated comfortably in the experiment room as detailed above. Experiment 2 was a speeded response task (Hershenson, 1962). A trial consisted of the presentation of a visual, auditory, or audiovisual stimulus with their temporal relationship as described above. Participants were asked to react as quickly as possible to any stimulus, auditory or visual, with a button press. Response time was recorded as the interval of time between stimulus onset (the first stimulus in 
429 the case of asynchronous audiovisual stimuli) and the button press. Experiment 3 was a

430 simultaneity judgement task (Zampini et al., 2005). Each trial consisted of the presentation of an

431 audiovisual stimulus with temporal relationships as described above. After each trial, a response

432 screen appeared, prompting participants to indicate on the number pad of a keyboard whether

433 each audiovisual pair were presented synchronously (by pressing "1") or not (by pressing “0”).

434 Participants were asked to take their time and answer as accurately as possible. For both

435 experiments, 20 trials of each condition were presented and trials were separated by a variable

436 time from $U(1,3)$.

437 Analysis

438 For Experiment 2, median response times (RTs) for auditory, visual, and audiovisual

439 presentations were calculated. The difference of median auditory and visual RTs was calculated.

440 A prediction of audiovisual RTs across SOA was computed by taking the minimum of each

441 auditory or visual RT while accounting for the lag imposed by the SOA (Hershenson, 1962).

442 Audiovisual RTs were subtracted from this prediction to yield a measure of RT facilitation.

443 These data were then fit to a gaussian curve:

444

$$
f(x)=a e^{\frac{(x-\mu)^{2}}{2 \sigma^{2}}}
$$

445 where $\mu$ represents the SOA at peak multisensory gain. For experiment 3 , the proportion of

446 synchronous judgement was computed at each SOA. These data were fit to a gaussian curve as

447 above, where the $\mu$ represents the point of subjective simultaneity (PSS) and $2 \sigma$ is a measure of

448 the width of the so-called temporal binding window.

449 Author Contributions

450 AN designed the experiment, collected and analyzed the data, and wrote the manuscript. AN,

$451 \mathrm{RR}$, and MW revised and approved the final version of the manuscript.

452 Acknowledgments

453 Support for this work was provided by NIH grant HD083211 to MTW.

454 Competing interests

455 The authors declare no competing interests. 


\section{References}

Atilgan, H., Town, S. M., Wood, K. C., Jones, G. P., Maddox, R. K., Lee, A. K. C., \& Bizley, J. K. (2018). Integration of Visual Information in Auditory Cortex Promotes Auditory Scene Analysis through Multisensory Binding. Neuron, 97(3), 640-655.e4. https://doi.org/10.1016/j.neuron.2017.12.034

Bizley, J. K., \& Cohen, Y. E. (2013). The what, where and how of auditory-object perception. Nature Reviews Neuroscience. https://doi.org/10.1038/nrn3565

Bizley, J. K., Jones, G. P., \& Town, S. M. (2016). Where are multisensory signals combined for perceptual decision-making? Current Opinion in Neurobiology. https://doi.org/10.1016/j.conb.2016.06.003

Bizley, J. K., Maddox, R. K., \& Lee, A. K. (2016). Defining Auditory-Visual Objects: Behavioral Tests and Physiological Mechanisms. Trends in Neurosciences, 39(2), 74-85. https://doi.org/10.1016/j.tins.2015.12.007

Blaser, E., Pylyshyn, Z. W., \& Holcombe, A. O. (2000). Tracking an object through feature space. Nature, 408(6809), 196-199. https://doi.org/10.1038/35041567

Brainard, D. H. (1997). The Psychophysics Toolbox. Spatial Vision, 10, 433-436. https://doi.org/10.1163/156856897X00357

Bregman, A. S. (1990). Auditory scene analysis : the perceptual organization of sound. MIT Press.

Carlyon, R. P. (2004). How the brain separates sounds. Trends in Cognitive Sciences. https://doi.org/10.1016/j.tics.2004.08.008

Chandrasekaran, C., Trubanova, A., Stillittano, S., Caplier, A., \& Ghazanfar, A. A. (2009). The natural statistics of audiovisual speech. PLoS Computational Biology, 5(7). https://doi.org/10.1371/journal.pcbi.1000436

Cherry, E. C. (1953). Some experiments on the recognition of speech, with one and with 2 ears. Journal of the Acoustical Society of America, 25(5), 975-979.

https://doi.org/10.1121/1.1907229

Chuen, L., \& Schutz, M. (2016). The unity assumption facilitates cross-modal binding of 
musical, non-speech stimuli: The role of spectral and amplitude envelope cues. Attention, Perception, and Psychophysics, 78(5), 1512-1528. https://doi.org/10.3758/s13414-016-10885

Crosse, M. J., Butler, J. S., \& Lalor, E. C. (2015). Congruent Visual Speech Enhances Cortical Entrainment to Continuous Auditory Speech in Noise-Free Conditions. Journal of Neuroscience, 35(42), 14195-14204. https://doi.org/10.1523/JNEUROSCI.1829-15.2015

Desimone, R., \& Duncan, J. (1995). Neural Mechanisms of Selective Visual Attention. Annual Review of Neuroscience, 18(1), 193-222. https://doi.org/10.1146/annurev.ne.18.030195.001205

Elhilali, M., Ma, L., Micheyl, C., Oxenham, A. J., \& Shamma, S. A. (2009). Temporal Coherence in the Perceptual Organization and Cortical Representation of Auditory Scenes. Neuron, 61(2), 317-329. https://doi.org/10.1016/j.neuron.2008.12.005

Elliott, T. M., \& Theunissen, F. E. (2009). The Modulation Transfer Function for Speech Intelligibility. PLoS Computational Biology, 5(3), e1000302. https://doi.org/10.1371/journal.pcbi.1000302

Feldman, J. (2003). What is a visual object? Trends in Cognitive Sciences. https://doi.org/10.1016/S1364-6613(03)00111-6

Grant, K. W., \& Seitz, P. F. P. (2000). The use of visible speech cues for improving auditory detection of spoken sentences. The Journal of the Acoustical Society of America, 108(3 Pt 1), 1197-1208. https://doi.org/10.1121/1.422512

Green, D. M., \& Swets, J. A. (1966). Signal detection theory and psychophysics. Society, 1, 521. https://doi.org/10.1901/jeab.1969.12-475

Griffiths, T. D., \& Warren, J. D. (2004). What is an auditory object? Nature Reviews Neuroscience, 5(11), 887-892. https://doi.org/10.1038/nrn1538

Henry, M. J., \& Obleser, J. (2012). Frequency modulation entrains slow neural oscillations and optimizes human listening behavior. Proceedings of the National Academy of Sciences, 109(49), 20095-20100. https://doi.org/10.1073/pnas.1213390109 
510

511

512

513

514

515

516

517

518

519

520

521

522

523

524

525

526

527

528

529

530

531

532

533

534

535

536

537

Hershenson, M. (1962). Reaction time as a measure of intersensory facilitation. Journal of Experimental Psychology, 63(3), 289-293. https://doi.org/10.1037/h0055703

Jack, C. E., \& Thurlow, W. R. (1973). Effects of degree of visual association and angle of displacement on the "ventriloquism" effect. Perceptual and Motor Skills, 37(3), 967-979. https://doi.org/10.2466/pms.1973.37.3.967

Kleiner, M., Brainard, D. H., Pelli, D. G., Broussard, C., Wolf, T., \& Niehorster, D. (2007). What's new in Psychtoolbox-3? Perception, 36, S14. https://doi.org/10.1068/v070821

Kösem, A., Gramfort, A., \& Van Wassenhove, V. (2014). Encoding of event timing in the phase of neural oscillations. NeuroImage, 92, 274-284. https://doi.org/10.1016/j.neuroimage.2014.02.010

Lee, S. H., \& Blake, R. (1999). Visual form created solely from temporal structure. Science, 284(5417), 1165-1168. https://doi.org/10.1126/science.284.5417.1165

Maddox, R. K., Atilgan, H., Bizley, J. K., \& Lee, A. K. (2015). Auditory selective attention is enhanced by a task-irrelevant temporally coherent visual stimulus in human listeners. ELife, 2015(4), 1-11. https://doi.org/10.7554/eLife.04995.001

Mesgarani, N., \& Chang, E. F. (2012). Selective cortical representation of attended speaker in multi-talker speech perception. Nature, 485(7397), 233-236. https://doi.org/10.1038/nature11020

Nan, Y., Liu, L., Geiser, E., Shu, H., Gong, C. C., Dong, Q., ... Desimone, R. (2018). Piano training enhances the neural processing of pitch and improves speech perception in Mandarin-speaking children. Proceedings of the National Academy of Sciences of the United States of America, 201808412. https://doi.org/10.1073/pnas.1808412115

Nidiffer, A. R., Diederich, A., Ramachandran, R., \& Wallace, M. T. (2018). Multisensory perception reflects individual differences in processing temporal correlations. Scientific Reports, 8(1). https://doi.org/10.1038/s41598-018-32673-y

O'Sullivan, J. A., Shamma, S. A., \& Lalor, E. C. (2015). Evidence for Neural Computations of Temporal Coherence in an Auditory Scene and Their Enhancement during Active Listening. Journal of Neuroscience, 35(18), 7256-7263. 
539

540

541

542

543

544

545

546

547

548

549

550

551

552

553

554

555

556

557

558

559

560

561

562

563

564

Parise, C. V., \& Ernst, M. O. (2016). Correlation detection as a general mechanism for multisensory integration. Nature Communications, 7(12), 364. https://doi.org/10.1038/ncomms11543

Parise, C. V., Spence, C., \& Ernst, M. O. (2012). When correlation implies causation in multisensory integration. Current Biology, 22(1), 46-49. https://doi.org/10.1016/j.cub.2011.11.039

Parise, C. V, Harrar, V., Ernst, M. O., \& Spence, C. (2013). Cross-correlation between Auditory and Visual Signals Promotes Multisensory Integration. Multisensory Research, 26, 1-10. https://doi.org/10.1163/22134808-00002417

Plass, J., Brang, D., Suzuki, S., \& Grabowecky, M. (2019). Vision Perceptually Restores Auditory Spectral Dynamics in Speech. Psyarxiv. https://doi.org/10.31234/OSF.IO/T954P

Shinn-Cunningham, B. G. (2008). Object-based auditory and visual attention. Trends in Cognitive Sciences, 12(5), 182-186. https://doi.org/10.1016/j.tics.2008.02.003

Simon, D. M., \& Wallace, M. T. (2017). Rhythmic Modulation of Entrained Auditory Oscillations by Visual Inputs. Brain Topography, 30(5), 565-578. https://doi.org/10.1007/s10548-017-0560-4

Teki, S., Chait, M., Kumar, S., Shamma, S., \& Griffiths, T. D. (2013). Segregation of complex acoustic scenes based on temporal coherence. ELife, 2, e00699. https://doi.org/10.7554/eLife.00699

Treisman, A. (1998). Feature binding, attention, and object perception. Essent. Sources Sci. Study Conscious., 8, 226. https://doi.org/10.1016/j.ejpn.2004.03.003

Vatakis, A., \& Spence, C. (2007). Crossmodal binding: evaluating the "unity assumption" using audiovisual speech stimuli. Perception \& Psychophysics, 69(5), 744-756. https://doi.org/10.3758/BF03193776

Vroomen, J., \& Stekelenburg, J. J. (2011). Perception of intersensory synchrony in audiovisual speech: Not that special. Cognition, 118(1), 78-86. 
565 https://doi.org/10.1016/j.cognition.2010.10.002

566 Wallace, M. T., \& Stevenson, R. A. The construct of the multisensory temporal binding

567 window and its dysregulation in developmental disabilities, 64 Neuropsychologia $§(2014)$.

568 https://doi.org/10.1016/j.neuropsychologia.2014.08.005

569 Wertheimer, M. (1923). Untersuchungen zur Lehre yon der Gestalt II. Psychologische Forschung, $570 \quad 4,301-350$.

571 Zampini, M., Guest, S., Shore, D. I., \& Spence, C. (2005). Audio-visual simultaneity

$572 \quad$ judgments. Perception E Psychophysics, 67(3), 531-544.

573 https://doi.org/10.3758/BF03193329

574 